\title{
Structural analysis of VDR complex with ZK168281 antagonist
}

\author{
Anna Y. Belorusova ${ }^{1-5^{*}}$, Sandra Chalhoub ${ }^{1-4}$, Daniela Rovito ${ }^{1-4}$, Natacha Rochel ${ }^{1-4^{*}}$ \\ 1. Institut de Génétique et de Biologie Moléculaire et Cellulaire (IGBMC), 67400 Illkirch, France \\ 2. Institut National de La Santé et de La Recherche Médicale (INSERM), U1258, 6740o Illkirch, France \\ 3. Centre National de Recherche Scientifique (CNRS), UMR7104, 67400 Illkirch, France \\ 4. Université de Strasbourg, 67400 Illkirch, France \\ 5. Medicinal Chemistry, Early Respiratory \& Immunology, BioPharmaceuticals R\&D, AstraZeneca, 43183 Gothen- \\ burg, Sweden
}

\begin{abstract}
Vitamin D receptor (VDR) antagonists prevent the VDR activation function helix 12 from folding into its active conformation, thus affecting coactivator recruitment and antagonizing the transcriptional regulation induced by 10,25-dihydroxyvitamin D3. Here we report the crystal structure of the zebrafish VDR ligand binding domain in complex with the ZK168281 antagonist, revealing that the ligand prevents optimal folding of the C-terminal region of VDR. This $\begin{array}{llllll}\text { interference was } & \text { confirmed } & \text { by } & \text { in } & \text { solution. }\end{array}$
\end{abstract}

\section{Introduction}

Ligand-induced transcription factors form a subgroup of the nuclear receptor superfamily. Agonist binding induces homo- or hetero-dimerization, the folding of the activation function helix $12(\mathrm{H} 12)$ into its active conformation to modulate coregulatory molecules recruitment, and DNA binding in target gene regulatory regions ${ }^{1}$. Antagonists, in turn, destabilize the nuclear receptor interaction with cofactors and repress transcription ${ }^{1}$. The receptor of the secosteroid hormone vitamin D (VDR), in complex with its heterodimeric partner RXR, regulate various physiological functions, such as calcium levels, cell growth and differentiation, apoptosis and adaptive/innate immune responses ${ }^{2-4}$. To modulate VDR activities, more than 4000 synthetic ligands have been developed by medicinal chemistry approaches ${ }^{5}$. Whereas most of them are agonists, very few elicit antagonistic properties ${ }^{6-}$ 8

Active VDR antagonists are defined as ligands preventing $\mathrm{H}_{12}$ from folding into its active conformation which in turn affects coactivator recruitment and antagonizes the transcriptional regulation induced by $1 \alpha, 25$-dihydroxyvitamin $\mathrm{D}_{3}$ (1,25 $\mathrm{D}_{3}$ ), the bioactive form of vitamin $\mathrm{D}_{3}{ }^{6-8}$. Analogs of the $\mathrm{ZK}$ series ${ }^{9-11}$ exhibit the skeleton of the clinically used vitamin $\mathrm{D}_{3}$ analog calcipotriol and possess a 25-carboxylic ester moiety in their side chain. Among them, only ZK168281, which exhibits the largest rigid chain (Figure $1 \mathrm{~A}$ ), has been described as a full VDR antagonist ${ }^{9-10}$. Modelling and molecular dynamics of ZK168281 docked into the VDR ligand binding pocket (LBP) by anchoring the 24-hydroxyl group predicted that it might prevent H12 from being correctly positioned in its active state ${ }^{10,12}$. However, no crystal structure of ZK168281 in complex with VDR was previously reported.

In the present study, we determined the crystal structure of the zebrafish (z) VDR ligand binding domain (LBD) in complex with ZK168281 and examined its conformational dynamics in solution by hydrogen/deuterium exchange coupled with mass spectrometry (HDX-MS). In addition, we investigated the potency of ZK168281 to antagonize 1,25D3-induced VDR activity in cells.

\section{Results}

\section{Binding mode of ZK168281 to zVDR LBD}

To determine the binding mode of ZK168281 in the VDR LBD, we carried out an X-ray crystallographic analysis of the zVDR LBD complexed with ZK168281. We attempted to crystallize VDR LBD/ZK168281 in absence or presence of the corepressor NCOR1 ID2 and ID1 (ASNLGLEDIIRKALMGS and RLITLADHICQIITQDFAR, respectively) or the coactivator SRC1. Due to weak affinity for corepressor peptides ${ }^{13}$ or non-optimal crystal packing, only crystals of the complex in the presence of the SRC 1 coactivator peptide NR2 (RHKILHRLLQEGS) were obtained and analyzed (Supplementary Table 1). These results are in agreement with previous reports characterizing X-ray structures of VDR complexes in presence of antagonist ligands ${ }^{14-19}$. 
A

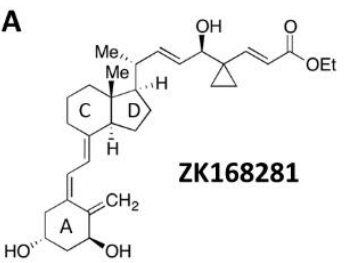

B

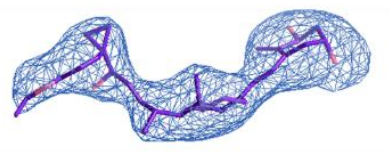

FIGURE 1: Binding mode of ZK168281. (A) Chemical structure of ZK168281 with A, C/D rings labelled. (B) ZK168281 modelled into the omit Polder map contoured at $3.5 \sigma$, with model bias reduction and exclusion of solvent density. (PDB ID: 6T2M)

ZK168281 binds to the VDR LBP unambiguously as shown by the POLDER omit map (Figure 1B). Superposition of the VDR LBP in the presence of $\mathrm{ZK}_{1} 68281$ or $1,25 \mathrm{D}_{3}$ shows that positioning of the A-ring of $\mathrm{ZK}_{168281}$ is similar to that of $1,25 \mathrm{D}_{3}$, but $\mathrm{C}_{1} \mathrm{D}_{\text {-rings }}$ of ZK168281 are slightly shifted (Figure $2 \mathrm{~A}$ ). The $1-\mathrm{OH}$ and $3-\mathrm{OH}$ groups in the A-ring of ZK168281 form hydrogen bonds similar to 1,25D3: with Ser265 and Arg302 and with Tyr175 and Ser3o6, respectively (Figure 2A,B). Note that the corresponding residues in human (h) and rat (r) VDR are listed in Supplementary Table 2. In contrast to the 25-OH group of $1,25 \mathrm{D}_{3}$ that forms specific hydrogen bonds with His333 and His423, a sole hydrogen bond with His423 is formed by the 24-OH group of ZK168281. Note that the carboxyl moiety of ZK168281 weakly interacts with His333 (Figure 2A). In addition to those residues, ZK168281 interacts with 15 residues of the LBP at a $4 \AA$ distance cutoff (Figure $2 \mathrm{~B}$ ) in comparison to 14 for $1,25 \mathrm{D}_{3}$. Differential interactions are observed for Alaz31 and Gly332 that interact with the terminal extended side chain of ZK168281 at $3.8 \AA$ and $4.0 \AA$, respectively (in contrast to 4.3 and $>5 \AA$ for $1.25 \mathrm{D}_{3}$ ) and Ala259 that selectively interacts with the cyclopropyl of ZK168281 at $3.7 \AA$. At the same time, ZK168281 forms weaker interaction with Leu255 at 4.1 $\AA$ (instead of $3.8 \AA$ for 1.25D3), Leu261 at $4.0 \AA$ (instead of $3.6 \AA$ for $1.25 \mathrm{D}_{3}$ ) and Leu337 at $4.9 \AA$ (instead of $3.7 \AA$ for $1.25 \mathrm{D}_{3}$ ). Importantly, Leu430 (H11) side chain is re-orientated in the presence of ZK168281 compared to that in $1,25 \mathrm{D}_{3}$-bound VDR LBD (Figure $2 \mathrm{C}$ ). To determine whether these differential interactions selectively modulate the VDR LBD conformation, we compared the overall 3D structures of ZK168281- and 1,25D3-bound VDR. Significant differences between two structures were revealed: the last four atoms of the ZK168281 side chain extend towards helix 11 and induce $\mathrm{C} \alpha$ shift for the last turn of helix H11 (1.2 $\AA$ ), the loop 11-12 (o.7 $\AA$ ) and helix H6 (o.7 $\AA$ ) (Figure $2 \mathrm{C}$ ). However, H12 adopts the agonist conformation in the presence of both compounds, and is involved in similar ligand interactions i.e. with $\mathrm{V} 444$ (Figure 2B).

Taken together, these results show that, whereas permitting H12 to adopt an active conformation, ZK168281 destabilizes the C-terminal region of VDR which could lead to its suboptimal agonistic conformation. Since the active fold could be explained by a large excess of coactivator peptide used to crystallize the complex and the crystal packing forces, we further probed antagonistinduced conformational changes of VDR in solution and assessed the effect of ZK168281 on H12 mobility using HDX-MS. 

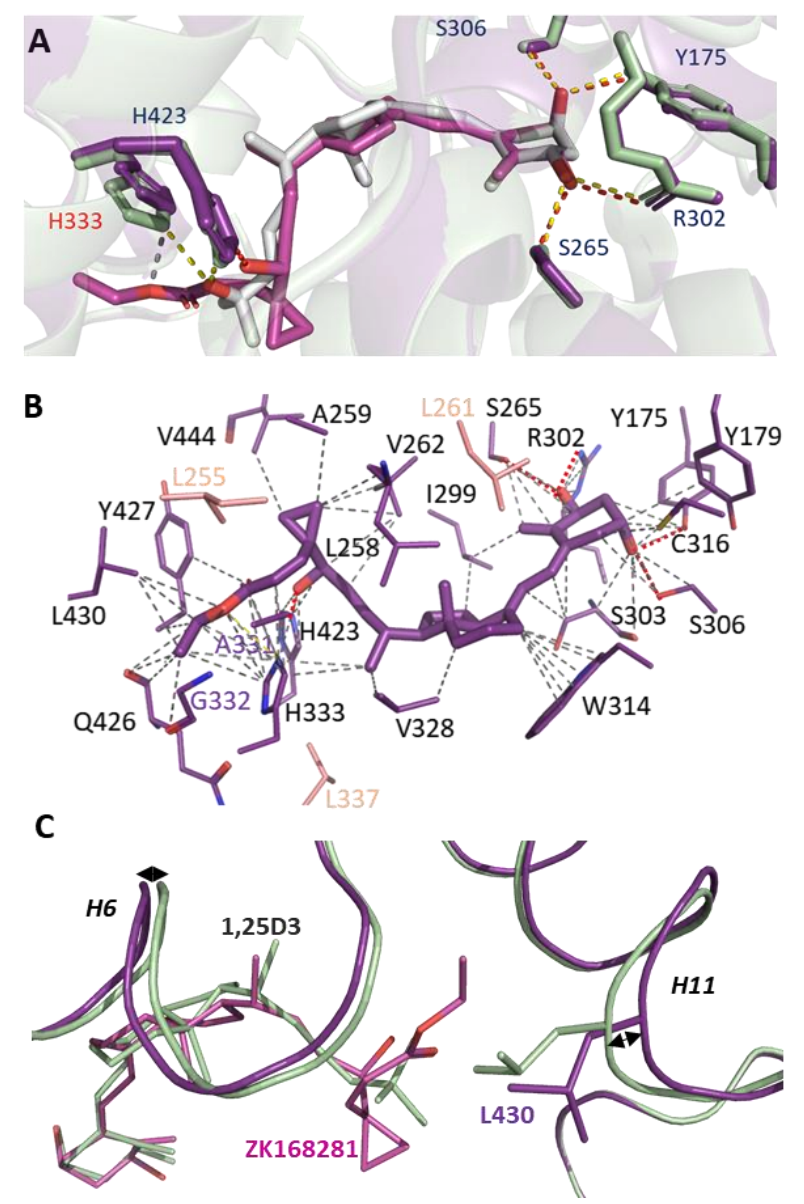

FIGURE 2: Structural accommodation of ZK168281 in the zVDR ligand binding pocket (PDB ID: 6T2M) (A) Hydrogen bonds interactions between 1,25D3 (yellow dashed lines) or ZK168281 (red dashed lines) with VDR. In ZK168281 complex, His333 does not form a hydrogen bond but interacts with the carbonyl moiety (grey dashed line). ZK168281 is shown in purple and 1,25D3 in grey. (B) Interactions between ZK168281 and residues lining the LBP of zVDR LBD at 4 Å cutoff. Differential interacting residues are shown in purple and salmon. (C) Conformational changes of $\mathrm{C} \alpha$ main chain and Leu430 side chain that opens an additional pocket to fit the ZK168281 side chain (purple) compared to VDR-1,25D3 (grey).

\section{HDX-MS analysis of 1,25D3- and ZK168281-zVDR complexes}

We achieved an excellent peptide coverage of $99 \%$ of zVDR LBD (Supplementary Figure 1) resulting in a high spatial resolution of the HDX-MS data. First, we characterized the conformational dynamics of unliganded (apo) zVDR LBD. H11 and H12 Cterminus, the insertion region (residues 186-251), $\beta$-sheets (residues $306-324$ ) and H6 (residues 325-330) of zVDR LBD exhibit rapid exchanges rate with the solvent, indicating that these regions are highly dynamic. In contrast, regions remote from the LBP and forming central part of the $\alpha$-helical sandwich, notably $\mathrm{H}_{4}$ (residues 286-295), $\mathrm{H}_{5}$ (residues 296-305), and H8-H9 (residues 355-399) are more protected from the solvent (Supplementary Figure 2). These observations are in line with those reported for human ${ }^{20}$ and rat $^{16}$ VDR. Interestingly, C-terminal Hio (residues $417-430$ ) showed $\sim 80 \%$ exchange to deuterium after 1 min, indicating that this region is very flexible in zebrafish in contrast to rat and human where it does not exceed $20 \%$ exchange after $60 \mathrm{~min}$.

We next analyzed the effect of $1,25 \mathrm{D}_{3}$ binding on zVDR LBD (Supplementary Figure $2 \mathrm{C}$ and Supplementary Figure $3 \mathrm{~A}$ ). A significant stabilization of structural elements forming the LBP ( $\left.\mathrm{H}_{1-2}, \mathrm{H}_{3}, \mathrm{H}_{5}, \mathrm{H}_{6}-7, \mathrm{H}_{10}-11\right)$ was observed, notably the regions involving residues forming hydrogen bonds with 1,25 $\mathrm{D}_{3}$ (i.e. Tyr175 of $\mathrm{H}_{1}$, Ser265 of $\mathrm{H}_{3}$, Arg302 and Ser3o6 of $\mathrm{H}_{5}$, His333 of the loop H6-H7 and His423 of Hul). Surprisingly, no protection from deuterium exchange was observed for zVDR H12 in tested conditions, indicating that $1,25 \mathrm{D}_{3}$ alone is not sufficient to fold $\mathrm{zH} 12$ in its active conformation, in contrast to rat and human VDR H12 that exhibit a large degree of protection upon agonist binding at similar time points ${ }^{16,20,21}$. This difference could be due to an increased intrinsic flexibility of the zebrafish VDR C-terminus observed for the apo form. HDX-MS experiments were next per-

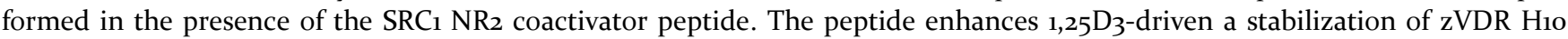
(residues 416-426) at 3 and 10 min exchange and leads to a large stabilization of zVDR H12 (residues 440-445) with 30-40\% increase in protection after 1 min exchange (Figure $3 \mathrm{~F}$, Supplementary Figure ${ }_{3} \mathrm{~B}$ ). Thus, despite its apparent flexibility, H12 of agonist-bound zVDR is stabilized upon coactivator binding. 
To investigate the effect of ZK168281 binding on the zVDR LBD conformation we performed differential HDX-MS analysis of the antagonist complex and compared it to $1,25 \mathrm{D}_{3} . \mathrm{H}_{3}$ and $\mathrm{H}_{7}$ regions displayed the highest protection (up to 6o-80\%) after ZK168281 binding when compared to apo states (Figure 3A,B and Supplementary Table 3). However, binding residues in $\mathrm{H}_{3}$ (residues 253-267), $\mathrm{H6}-\mathrm{H}_{7}$ (residues 322-344) and $\mathrm{H}_{10}-\mathrm{H}_{11}$ (residues 416-437) were 10-15\% less protected in comparison to 1,25 $\mathrm{D}_{3}$ (Figure ${ }_{3}$ C,D and Supplementary Table 2 ). The stabilization of residues of $\mathrm{H}_{1}$ and $\mathrm{H}_{2}$ by ZK168281 was similar to $1,25 \mathrm{D}_{3}$ (Figure $3 \mathrm{D}$ ). In addition, $\mathrm{H}_{5}$ (residues $290-297$ ) showed more than $30 \%$ of deprotection compared to $1,25 \mathrm{D}_{3}$ (Figure ${ }_{3} \mathrm{C}$-D and Supplementary Figure 4A,B). As Lys292 $\left(\mathrm{H}_{5}\right)$ is forming hydrogen bond with the backbone NH of Gly449 (H12), these results indicate that ZK168281 fails to fold zVDR H12 into its active conformation. Importantly, the presence of SRC-1 NR2 peptide does not enhance ZK168281-induced zVDR LBD stabilization, including H12 (Figure 3E,F, Supplementary Figure 2D and Supplementary Figure $3 C, D)$. Altogether, differential HDX-MS data show that, contrary to 1,25D3, ZK168281 binding does not lead to the stabilization of active H12 conformation and prevents zVDR-coactivator interaction.

This impaired interaction between zVDR-ZK168281 and SRC1 peptide was further confirmed by measurement of the affinity of $S_{1} C_{1} N_{2}$ peptide for zVDR LBD in solution by MicroScale Thermophoresis. In the presence of saturating $1,25 D_{3}$ concentration, the $\mathrm{SRC}_{1}$ peptide was efficiently recruited by $\mathrm{zVDR}(\mathrm{Kd} 1.2 \mu \mathrm{M})$ in agreement with published data ${ }^{13}$. In contrast, the recruitment of $\mathrm{SRC}_{1}$ by ZK168281-liganded zVDR LBD was strongly impaired (Kd >30 $\mu \mathrm{M}$; Supplementary Figure $4 \mathrm{C}$ ). Similar results were obtained for the full-length hVDR/RXR $\alpha$ heterodimer (Supplementary Figure $4 \mathrm{D}$ ).

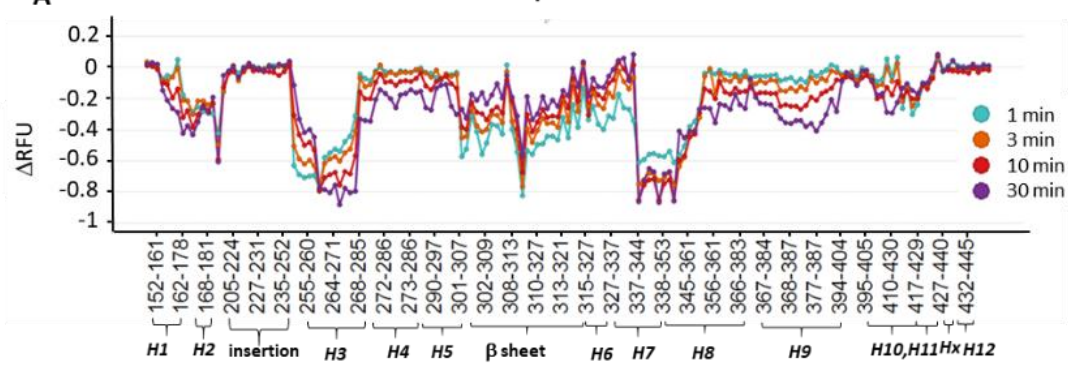

D

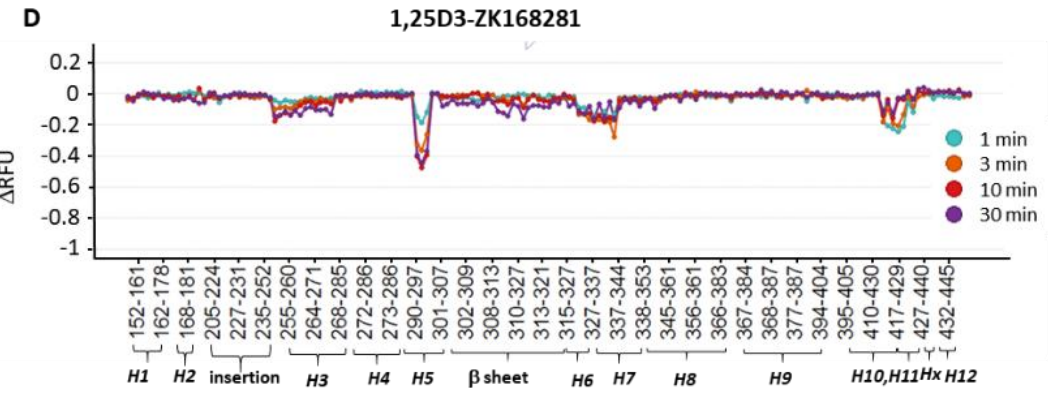

B

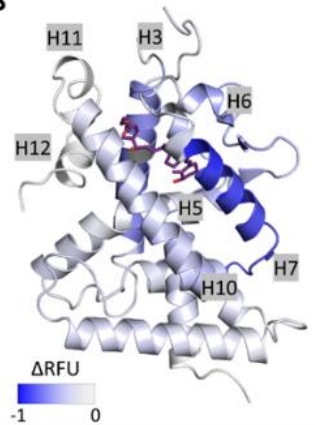

$\mathbf{E}$

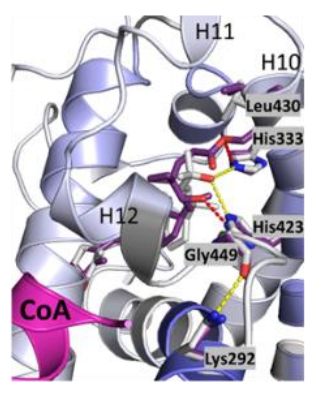

C

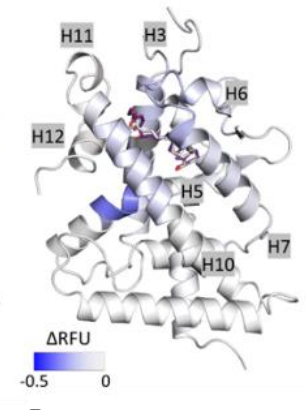

$\mathbf{F}$

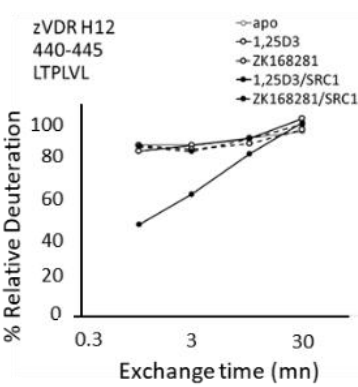

FIGURE 3: HDX analysis of the ZK168281-bound zVDR LBD. (A) Relative fractional uptake (RFU) difference plot showing the variations of deuterium uptake between apo- and ZK168281-bound zVDR LBD. A negative difference value corresponds to a protection from deuterium uptake upon ligand binding, with large absolute values corresponding to a strong effect. (B) Protection from deuterium exchange upon ZK168281 binding mapped onto ZK168281-zVDR crystal structure, RFU levels are the average of four time points (1, 3, 10, 30 min). Regions of the zVDR structure not covered by HDX-MS are shown in dark grey. (C) Average RFU difference mapped onto ZK168281-zVDR crystal structure (PDB: 6T2M) and corresponding RFU difference plot showing the variations of deuterium uptake between 1,25D3- and ZK168281-bound zVDR LBD (D). Regions of the zVDR structure not covered by HDX-MS are shown in dark grey. (E) Details of the interactions between ZK168281 (purple) and zVDR LBP (CA-atoms colored according to the difference in RFU between 1,25 $\mathrm{D}_{3}$ and $\mathrm{ZK} 168281$ measured in the presence of the coactivator SRC1). (F) Deuterium uptake of the LTPLVL peptide from zVDR H12 (residues 440-445) in different complexes plotted as a function of time. Deuterium uptake values for all peptides were corrected to back exchange using the fully deuterated control. HDX-MS measurements were performed in triplicates. Full peptide list and statistical summary are presented in the Supplementary Table 2.

\section{ZK168281 antagonizes VDR transcriptional activity.}

To characterize antagonistic activities of ZK168281, VDR reporter gene assays were performed in the human embryonic kidney cell line HEK293 EBNA with hVDR. Cells were transfected with expression vectors encoding the full-length hVDR and a luciferase reporter plasmid encompassing the well-characterized promoter region of the main human VDR target gene $C Y P_{24} A_{1}{ }^{22}$, and treated with $10 \mathrm{nM} 1,25 \mathrm{D}_{3}$ in the presence of various ZK168281 concentrations for $18 \mathrm{~h}$ (Figure $4 \mathrm{~A}$ ). 1,25 $\mathrm{D}_{3}$ increased luciferase activity by 50 -fold compared to vehicle-treated cells (Figure $4 \mathrm{~A}$ ), and ZK168281 reduced 1,25D3-induced reporter activity by up to $60 \%$ in a dose-dependent manner. To further determine if ZK168281 similarly antagonizes zVDR activity, we performed a similar cell-based VDR reporter gene assay in rat ileum derived cell line (IEC-18) using full-length zVDR and human CYP24A1 luciferase reporter plasmid (Figure $4 \mathrm{~B}$ ). While, ${ }_{1,25} \mathrm{D}_{3}$ increased reporter activity in IEC-18 at $100 \mathrm{nM}$ concentration (Figure $4 \mathrm{~B}$ ), ZK168281 reduced zVDR mediated ${ }_{1,25} \mathrm{D}_{3}$-induced activity at any tested dose (Figure $4 \mathrm{~B}$ ). Thus, ZK168281 similarly antagonizes $1,25 \mathrm{D}_{3}-$ induced hVDR and zVDR activity. 

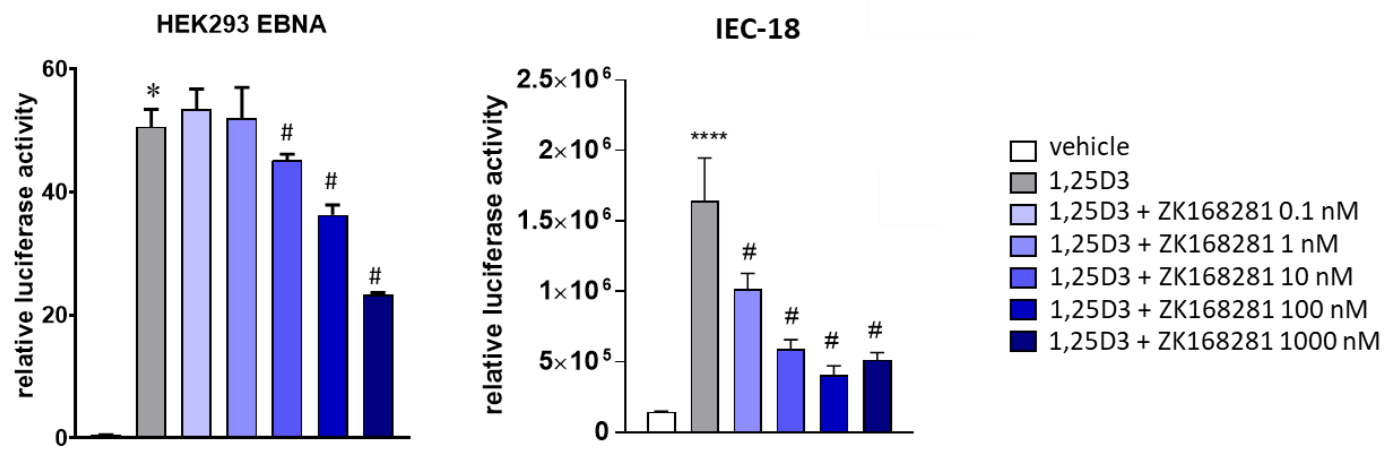

FIGURE 4: Effect of ZK168281 on hVDR and zVDR transcriptional activity. Luciferase gene assay performed in HEK293 EBNA cells transfected with expression vectors encoding the full-length hVDR and a human $C Y P_{2} 4 A_{1}$ luciferase reporter plasmid, and treated for $18 \mathrm{~h}$ with vehicle or $10 \mathrm{nM} 1,25 \mathrm{D}_{3}$ in the presence or absence of the indicated ZK168281 doses (o.1 to $1000 \mathrm{nM}$ ) (B) IEC18 cells zVDR transfected with expression vectors encoding the full-length zVDR and a CYP24A1 luciferase reporter plasmid, and treated for $24 \mathrm{~h}$ with vehicle or different ZK168281 doses ( 1 to $1000 \mathrm{nM}$ ) in the presence of $100 \mathrm{nM}$ of $1,25 \mathrm{D}_{3}$. $\mathrm{n}=3{ }^{*} \mathrm{p}<0.05$ vs. vehicle, \# $\mathrm{p}<0.05$ vs. $1,25 \mathrm{D}_{3}$.

\section{Discussion and Conclusions}

In the present study, we determined the conformational changes of VDR LBD induced by ZK168281 and show that this vitamin $\mathrm{D}$ analog fails to stabilize zVDR H12 folding and to recruit co-activator peptide which provides basis for its antagonistic properties. Furthermore, we show that ZK168281 antagonizes both hVDR and zVDR cellular transcriptional activities.

We solved the crystal structure of zVDR LBD in complex with the antagonist ZK168281 revealing that insufficient stabilization of the C-terminal region of VDR results from ZK168281-induced shifts of H11, loop 11-12 and H6, the repositioning of Leu430, the loss of a hydrogen bond with His333, and of the interaction with Leu337 previously described as key amino acid for VDR agonism $^{22}$. The observed structural rearrangement slightly differs from the initial modeling ${ }^{10,12}$, that proposed either a perturbation of hHis397 (zHis423) and hPhe422 (zPhe448) interaction ${ }^{10}$ or a steric hindrance with hAla231 (zAla259) and hVal418 $(\mathrm{zVal} 444)^{12}$. The suboptimal positioning of helix H11 and the loop 11-12 does not prevent the active fold of H12 observed in the crystal structure. However, the crystal structure of the complex was obtained in the presence of excess of coactivator peptide, underlying the necessity to use other methods to investigate the antagonist binding mode, such as HDX-MS, a pseudo-structural method detecting perturbations in protein dynamics in solution. Indeed, HDX-MS confirmed the destabilization of the Cterminal region by ZK168281 suggested by the crystal structure, and additionally identified helix $\mathrm{H}_{5}$ as the most affected region in the antagonist-bound zVDR complex in comparison with $1,25 \mathrm{D}_{3}$. This differs from the reported HDX-MS analysis of rVDR in complex with the 25-dibenzyl antagonist which also prevents the VDR LBD C-terminus from folding into an active state however does not impact $\mathrm{H}_{5}{ }^{16}$.

Here we show that albeit the structural dynamics of zVDR LBD in its apo form is similar to previously characterized rVDR ${ }^{16}$ and $\mathrm{hVDR}^{20}$ LBDs, its C-terminus exhibits larger flexibility. H12 of zVDR remains flexible upon agonist binding in solution; similar behavior was described for hRXR bound to 9cisRA or hER bound to estradiol. ${ }^{20}$ Nevertheless, in the presence of the SRC1 NR2 coactivator peptide, a large stabilization effect was observed on agonist-bound zVDR H12 similarly to hVDR ${ }^{21}$. Similar structural elements were stabilized upon $1,25 \mathrm{D}_{3}$ binding. Thus, in agreement with our previous analysis comparing ligand binding modes of $1,25 \mathrm{D}_{3}$ between $\mathrm{hVDR}$ and $\mathrm{zVDR}{ }^{23}$, these results confirmed the validity of the use of zVDR LBD for structural studies of complexes with agonist ligands and coactivator peptides. As demonstrated in this work, due to artefact crystal packing of antagonist bound VDR complexes, a full understanding of the structural consequences of antagonist binding could only be obtained by combining $x$-ray crystallography with solution structural methods.

Several crystal structures of VDR LBD complexes with antagonists have been previously reported, all crystalized in presence of a coactivator peptide ${ }^{14-19}$. Among them, the complexes with adamantyl $1^{14}$ and $22 \mathrm{~S}$-butyl combined with $25 \mathrm{p}^{-h y d r o x y p h e n y} \mathrm{l}^{17}$. Together with the structure reported here, crystal structures of active VDR antagonists confirm that these ligands destabilize the C-terminal region of the receptor but via different mechanisms (Supplementary Figure 5). ZK168281 induces the shifts of Hi1, loop 11-12 and H6, the repositioning of zLeu430(hLeu404) and the loss of a hydrogen bond with zHis333 (hHis305). The 25adamantyl-25-hydroxyl analog maintains the H-bonds of 1,25 $\mathrm{D}_{3}$ but induces a widening of the LBD around the H11, loop $11-12$ and $\mathrm{N}$-terminal $\mathrm{H}_{3}$ with side chain conformational changes of rLeu40o(hLeu404) and rLeu410(hLeu414). The 25-hydroxyphenyl analog induces the largest structural difference with a modified positioning of helix 11 and loop11-12 due to a steric repulsion. In addition, the rHis301(hHis305) and rHis393(hHis397) do not form hydrogen bonds.

VDR antagonists have a high therapeutic potential to treat diseases associated with VDR hyperfunction ${ }^{24}$. The potency of ZK168281 to reduce $1,25 \mathrm{D}_{3}$-induced gene expression has previously been shown to vary among the genetically engineered cancerderived immortalized cell lines (Cos-7, Saos-2, HaCaT and MCF-7) overexpressing VDR ${ }^{11}$. In the present study, we show that ZK168281 blunts 1,25D3-induced hVDR and zVDR transcriptional activity, thus acting as a non-species specific VDR antagonist. 
ZK168281 is a good potential candidate for diseases associated with VDR hyperfunction, and this work provides atomic details of its binding mode as well as its effect on VDR conformational dynamics in solution that could be used for a rational design of next generation antagonists of VDR.

\section{Experimental section}

\section{Chemicals}

Compounds 1,25D3 (17936, Sigma Aldrich) and ZK1682819 (gift from the Medicinal Chemistry Department of Schering AG, now Bayer) were dissolved in absolute ethanol at $10^{-2} \mathrm{M}$ and stored at $-20{ }^{\circ} \mathrm{C}$. The fluorescent $\mathrm{SRC}_{1} \mathrm{NR}_{2}$ peptide (683LTERHKILHRLLQEGSPSD-701) was purchased from Thermo-Fisher. The SRC1 NR2 peptide (686-RHKILHRLLQEGSPS-70o) was synthesized by Pascal Eberling (IGBMC peptide synthesis common facility).

\section{Cells}

IEC-18 rat intestinal epithelial cells (American Type Culture Collection, Rockville, USA) were grown in Dulbecco's modified Eagle's medium (DMEM) $4.5 \mathrm{~g} / 1$ glucose supplemented with $5 \%$ fetal calf serum (FCS), 1 mM Sodium Pyruvate, o.1 UI/mL insulin and $40 \mu \mathrm{g} / \mathrm{mL}$ gentamicin. HEK293 EBNA human embryonic renal cells were maintained in DMEM supplemented with $10 \%$ FCS. Cells at $80 \%$ confluency were grown in medium supplemented with $5 \%$ charcoal-treated FCS for 48 h, and treated as indicated.

\section{Luciferase assay Transactivation Assays}

Cells were seeded into 24-well plates $\left(5 \times 10^{4}\right.$ cells per well). HEK293 EBNA cells were co-transfected with 150 ng of the expression plasmid $\mathrm{pSG}_{5}$-hVDR wild-type and $150 \mathrm{ng}$ of the reporter plasmid pLuc-MCS (Stratagene, La Jolla, USA) containing the proximal promoter region (-414 to -64) of the human CYP24A1 gene, 3 ng of the pRL plasmid (Promega, Madison, USA) containing the Renilla luciferase gene, and $697 \mathrm{ng}$ of the carrier plasmid pBluescript (Stratagene). Transfection was performed with jetPEI (PolyPlus Transfection, Illkrich, France) according to the manufacturer's instructions. IEC18 cells were co-transfected with an expression vector for human VDR (hVDR, $0.5 \mu \mathrm{g}$ per well) and zebrafish VDR (zVDR, o.5 $\mu$ g per well) respectively, a plasmid containing the proximal promoter region $(-414$ to -64$)$ of the human $C Y P_{24} A_{1}$ gene linked with the firefly luciferase reporter gene (cyp24-RE, $0.25 \mu \mathrm{g}$ per well), and a plasmid expressing $\beta$-galactosidase ( $\beta$-gal, $0.25 \mu \mathrm{g} /$ well) using X-tremeGENE HP DNA Transfection Reagent (Roche Diagnostics) following the manufacturer's instructions.

Six hours after transfection, tested compounds were added. Cells were harvested after $18 \mathrm{~h}$ or $24 \mathrm{~h}$ of incubation with ligands for hVDR and zVDR, respectively. Cells were lysed (125mM Tris-Phospahate pH 7.8, 10 mM EDTA, 5 mM DTT, 50\% Glycerol, 5\% triton $\mathrm{X}-100)$ and $20 \mu \mathrm{l}$ of lysates were assayed for luciferase activity as recommended by the supplier (Perkin-Elmer) and the Renilla luciferase or $\beta$-gal activity was used to normalize the transfection efficiencies.

\section{Crystallization and Structure Determination}

Expression of zebrafish VDR LBD (residues 156-453) and the following purification were done by the procedure reported previously $^{25}$. The protein was concentrated using Amicon ultra-30 (Millipore) to $3-7 \mathrm{mg} / \mathrm{ml}$ and incubated with a two-fold excess of ligand and a three-fold excess of the coactivator SRC-1 NR2 peptide. Crystals were obtained in 50 mM Bis-Tris pH 6.5, $1.6 \mathrm{M}$ lithium sulfate and $50 \mathrm{mM}$ magnesium sulfate. Protein crystals were mounted in a fiber loop and flash-cooled under a nitrogen flux after cryo-protection with $20 \%$ glycerol. Data collection from a single frozen crystal was performed at $100 \mathrm{~K}$ on the IDzoB beamline at ESRF (France). The raw data were processed with XLS ${ }^{26}$ and scaled with AIMLESS ${ }^{27,28}$ programs. The crystals belong to the space group $\mathrm{P6}_{5} 22$, with one LBD complex per asymmetric unit. The structure was solved and refined using BUSTER ${ }^{29}$, Phenix $^{30}$ and iterative model building using COOT ${ }^{31}$. Crystallographic refinement statistics are presented in Supplementary Table 1. All structural figures were prepared using PyMOL (www.pymol.org/).

\section{HDX-MS}

Prior the deuterium incorporation experiments, $10 \mu \mathrm{M}$ zVDR LBD protein was incubated with ten-fold excess of ligand $\left(1,25 \mathrm{D}_{3}, \mathrm{ZK} 168281\right)$ or equal volume of ethanol as a control for $30 \mathrm{~min}$ at room temperature. In the experiments with the coactivator peptide fifteen-fold excess of RHKILHRLLQEGS peptide from the human SRC1 receptor-interacting domain 2 (Thermo Fisher) was added to the protein-ligand complex. A fully deuterated zVDR LBD sample was generated by incubating protein with $4 \mathrm{M}$ Guanidine- $\mathrm{HCl}$ in $\mathrm{D}_{2} \mathrm{O}$ for $30 \mathrm{~min}$ at $45^{\circ} \mathrm{C}$. Exchange reactions were performed with a CTC PAL sample handling robot (LEAP Technologies). Reactions were conducted by incubating $4 \mu$ of protein for 6o, 180, 60o or $1800 \mathrm{~s}$ with $50 \mu \mathrm{l}$ of $\mathrm{D}_{2} \mathrm{O}$ buffer (50 mM Tris $\mathrm{pH}$ 8.0, $50 \mathrm{mM} \mathrm{NaCl}, 2 \%$ Glycerol, $1 \mathrm{mM}$ TCEP). The exchange reaction was quenched by the addition of $50 \mu$ of $3 \mathrm{M}$ Urea and o.1\% TFA, followed by immediate injection into a protease column for protein digestion $\left(2 \mathrm{~min}\right.$ at $\left.24^{\circ} \mathrm{C}\right)$. Enzymate BEH Pepsin $2.1 \times 30 \mathrm{~mm}$ Column (Waters, U.K.) was used. Peptic digest was further injected on a Waters nanoACQUITY UPLC System where peptides were first desalted by trapping for 3 min on a VanGuard Pre-Column Acquity UPLC BEH C18 $(1.7 \mu \mathrm{m} ; 2.1 \times 5 \mathrm{~mm}$ ), and then eluted over 6-min 5-40\% (vol/vol) acetonitrile (containing o.1\% formic acid) gradient into a Waters Synapt G2-Si mass spectrometer. Peptide separation was conducted at o.1 ${ }^{\circ} \mathrm{C}$. All exchange reactions were performed in triplicates.

Peptides from three samples of non-deuterated sample were identified using the ProteinLynx Global Server (PLGS Waters, U.K.). Peptides with an intensity of over 5000, a mass error $<5 \mathrm{ppm}$ and present in at least two of the three data acquisitions were pooled and imported into the DynamX data analysis software (Waters, U.K.). After a first round of automated spectral processing using DynamX, each peptide was inspected manually for suitability for further analysis. For zVDR LBD, a total of 164 peptides remained, with $99 \%$ coverage and a mean redundancy of 6.427 per amino acid. For all peptides, a value of relative ex- 
change per amino acid was corrected to back exchange by using the fully deuterated controls. The mean deuteration level per amino acid was calculated using Matlab (Mathworks) according to ${ }^{32}$ and subsequently mapped onto the crystal structures with PyMOL (Schrodinger LLC). For the representation of the apo zVDR LBD comprising insertion region, loop modelling with MOE (Chemical Computing Group Inc.) was applied on $2 \mathrm{HC} 4$.pdb file.

\section{Microscale thermophoresis}

Measurements were performed with a Monolith NT.115 instrument (NanoTemper Technologies GmbH, Munchen, Germany) monitoring the binding of fluorescein labelled SRC1 NR2 peptide to zVDR LBD or hVDR full-length/hRXR $\alpha \Delta \mathrm{NTD}$. The hVDR full-length/hRXR $\alpha \Delta$ NTD complexes were purified as described in ${ }^{33}$ followed by a final size exclusion chromatography step on a Superdex 200 (GE) column equilibrated in $20 \mathrm{mM}$ Hepes pH 7.5, $200 \mathrm{mM} \mathrm{NaCl}$, Glycerol 2\%, 1 mM TCEP, o.o5\% Tween 20. Each measurement consisted of 16 reaction mixtures where the fluorescent-labeled peptide concentration was kept constant (100 nM) and hVDR/RXR was serially diluted from a concentration of $10 \mu \mathrm{M}$ down to $0.5 \mathrm{nM}$. Measurements were performed in standard glass capillaries (Nanotemper) at $25^{\circ} \mathrm{C}$, at $15-20 \%$ LED excitation and $20 \%$ MST power, with a laser-on time of 30 s and a laser-off time of $5 \mathrm{~s}$.

For zVDR, the fluorescent-labeled peptide concentration was $50 \mathrm{nM}$ and zVDR concentrations comprised between $14 \mu \mathrm{M}$ and $0.4 \mathrm{nM}$. Measurements were made with standard glass capillaries (Nanotemper) at $25^{\circ} \mathrm{C}$, at $50 \%$ LED excitation and $40 \%$ MST power, with a laser-on time of $30 \mathrm{~s}$ and a laser-off time of $5 \mathrm{~s}$.

NanoTemper Analysis 2.2.4 software was used to fit the data and to determine the $K_{D}$.

\section{Statistics}

Transactivation assays data are shown as the mean of assays performed in triplicate \pm standard deviation. Statistical comparisons of data from three experimental groups were made by one-way ANOVA and were considered to be statistically significant if $\mathrm{p}<0.05$, and are indicated by an asterisk in the figures (GraphPad Software Prism 7). For the HDX-MS data, MEMHDX ${ }^{34}$ was used to identify statistically significant changes of deuterium uptake that occurred on compound binding (http://memhdx.czbi.pasteur.fr/).

\section{ASSOCIATED CONTENT}

Accession Code. The atomic coordinates and structure factors have been deposited accession code 6T2M. Authors will release the atomic coordinates and experimental data upon article publication.

\section{Supporting Information.}

Table of the refinement of the crystal structure as well as ZK168281 HPLC traces, additional supplementary figures and $3 \mathrm{D}$ model of full zVDR LBD (PDB) can be found in SI appendix (PDF).

\section{AUTHOR INFORMATION}

\section{Corresponding Authors}

* A.Y. Belorusova (anna.belorusova@astrazeneca.com)

or N. Rochel (rochel@igbmc.fr)

ORCID

Anna Y Belorusova: oooo-0oo2-5229-4536

Natacha Rochel: oooo-0oo2-3573-5889

\section{Author Contributions}

A.Y.B. and N.R conceived the study. A.Y.B produced protein, solved the crystal structure, performed and analyzed HDX-MS. S.C. produced proteins and performed MST experiments. A.Y.B., S.C. and D.R. performed cellular assays. A.Y.B. and N.R. wrote the manuscript.

\section{ACKNOWLEDGMENT}

We acknowledge the Medicinal Chemistry Department of Schering AG, now Bayer, for the ZK168281 compound. We thank the IGBMC staff: cell culture service, P. Eberling for peptide synthesis, A. McEwen for help in X-ray data collections, and C. PelusoIltis for excellent technical assistance. The authors thank the staff of IDzo at ESRF for assistance in using the beamlines. We thank Gilles Laverny (IGBMC) for discussion. The work was supported by grant ANR-13-BSV8-0024-01 from ANR and institutional funds from Instruct-ERIC for support and the use of resources of the French Infrastructure for Integrated Structural Biolo- 
gy (ANR-10-LABX-0030-INRT and ANR-10-IDEX-0oo2-02), Alsace contre le Cancer, the Fondation pour la Recherche Médicale (FRM-FDT20140930978). A.Y.B. is a fellow of the AstraZeneca R\&D postdoc program. S.C. thanks ARTP for a doctoral fellowship.

\section{ABBREVIATIONS}

1,25 $\mathrm{D}_{3}, 1 \alpha, 25$-dihydroxyvitaminD3; VDR, vitamin D receptor; LBD, ligand binding domain: LBP, ligand binding pocket; HDX-MS, hydrogen-deuterium exchange mass spectrometry.

\section{REFERENCES}

(1) Burris, T.P.; Solt, L.A.; Wang, Y.; Crumbley, C.; Banerjee, S.; Griffett, K.; Lundasen, T.; Hughes, T.; Kojetin, D.J. Nuclear receptors and their selective pharmacologic modulators. Pharmacol Rev. 2013, 65, 710-778.

(2) Christakos, S.; Dhawan, P.; Verstuyf, A.; Verlinden, L.; Carmeliet, G. Vitamin D: metabolism, molecular mechanism of action, and pleiotropic effects. Physiol Rev. 2016, 96, 365-408.

(3) Fleet, J.C. The role of vitamin D in the endocrinology controlling calcium homeostasis. Mol Cell Endocrinol 2017, 453, 36-45.

(4) Bikle, D.D. Vitamin D: newer concepts of its metabolism and function at the basic and clinical level. J Endocr Soc. 2020, 4, bvz038.

(5) Maestro, M.A.; Molnár, F.; Carlberg, C. Vitamin D and its synthetic analogs. J Med Chem. 2019, 62, 6854-6875.

(6) Mutchie, T.R.; Yu, O.B.; Di Milo, E.S. ; Arnold, L.A. Alternative binding sites at the vitamin D receptor and their ligands. Mol Cell Endocrinol. 2019, 485, 1-8.

(7) Saitoh, H. Vitamin D receptor antagonists. Vitamin D: Fourth Edition. 2017, 679-694.

(8) Belorusova, A.Y.; Rochel, N. Structural basis for ligand activity in vitamin D receptor. Vitamin D: Fourth Edition. $2017,189-209$.

(9) Bury, Y.; Steinmeyer, A.; Carlberg, C. Structure activity relationship of carboxylic ester antagonists of the vitamin D(3) receptor. Mol Pharmacol. 2000, 58, 1067-1074.

(10) Väisänen, S.; Peräkylä, M.; Kärkkäinen, J.I.; Steinmeyer, A.; Carlberg, C. Critical role of helix 12 of the vitamin D(3) receptor for the partial agonism of carboxylic ester antagonists. J Mol Biol. 2002, 315, 229-238.

(11) Herdick, M.; Steinmeyer, A.; Carlberg, C. Carboxylic ester antagonists of ralpha,25-dihydroxyvitamin D(3) show cell-specific actions. Chem Biol. 2000, 7,885-894.

(12) Tochini-Valentini, G.; Rochel, N.; Wurtz, J.M.; Moras, D. Crystal structures of the vitamin D nuclear receptor liganded with the vitamin D side chain analogues calcipotriol and seocalcitol, receptor agonists of clinical importance. Insights into a structural basis for the switching of calcipotriol to a receptor antagonist by further side chain modification. J Med Chem. 2004, 47, 1956-1961.

(13) Teichert, A. ; Arnold, L.A. ; Otieno, S.; Oda, Y.; Augustinaite, I.; Geistlinger, T.R.; Kriwacki, R.W.; Guy, R.K.; Bikle, D.D. Quantification of the vitamin D receptor-coregulator interaction. Biochemistry. 2009, 48, 1454-1461.

(14) Nakabayashi, M.; Yamada, S.; Yoshimoto, N.; Tanaka, T.; Igarashi, M.; Ikura, T.; Ito, N.; Makishima, M.; Tokiwa, H.; DeLuca, H.F.; Shimizu, M. Crystal structures of rat vitamin D receptor bound to adamantyl vitamin D analogs: structural basis for vitamin D receptor antagonism and partial agonism. J Med Chem. 2008, 51, 5320-5329.

(15) Inaba, Y.; Yoshimoto, N.; Sakamaki, Y.; Nakabayashi, M.; Ikura, T.; Tamamura, H.; Ito, N.; Shimizu, M.; Yamamoto, K. A new class of vitamin D analogues that induce structural rearrangement of the ligand-binding pocket of the receptor. J Med Chem. 2009, 52, 14381449.

(16) Kato, A.; Itoh, T.; Anami, Y.; Egawa, D.; Yamamoto, K. Helix12-stabilization antagonist of vitamin D receptor. Bioconjug Chem. 2016, 27, 1750-1761.

(17) Kato, A.; Yamao, M.; Hashihara, Y.; Ishida, H.; Itoh, T.; Yamamoto, K. Vitamin D analogues with a p-hydroxyphenyl group at the C25 position: crystal structure of vitamin D receptor ligand-binding domain complexed with the ligand explains the mechanism underlying full antagonistic action. J Med Chem. 2017, 6o, 8394-8406.

(18) Asano, L.; Waku, T.; Abe, R.; Kuwabara, N.; Ito, I.; Yanagisawa, J.; Nagasawa, K.; Shimizu, T. Regulation of the vitamin D receptor by vitamin D lactam derivatives. FEBS Lett. 2016, 590, 3270-3279.

(19) Anami, Y.; Itoh, T.; Egawa, D.; Yoshimoto, N.; Yamamoto, K. A mixed population of antagonist and agonist binding conformers in a single crystal explains partial agonism against vitamin D receptor: active vitamin D analogues with 22R-alkyl group. J Med Chem. 2014, $57,4351-4367$.

(20) Zhang, J.; Chalmers, M.J.; Stayrook, K.R.; Burris, L.L.; Garcia-Ordonez, R.D.; Pascal, B.D.; Burris, T.P.; Dodge, J.A.; Griffin, P.R. Hydrogen/deuterium exchange reveals distinct agonist/partial agonist receptor dynamics within vitamin $\mathrm{D}$ receptor/retinoid $\mathrm{X}$ receptor heterodimer. Structure. 2010, 18, 1332-1341.

(21) Zheng, J.; Chang, M.R.; Stites, R.E.; Wang, Y.; Bruning, J.B.; Pascal, B.D.; Novick, S.J.; Garcia-Ordonez, R.D.; Stayrook, K.R.; Chalmers, M.J.; Dodge, J.A.; Griffin, P.R. HDX reveals the conformational dynamics of DNA sequence specific VDR co-activator interactions. Nat Commun. 2017, 8, 923.

(22) Huet, T.; Laverny, G.; Ciesielski, F.; Molnár, F.; Ramamoorthy, T.G.; Belorusova, A.Y.; Antony, P.; Potier, N.; Metzger, D.; Moras, D.; Rochel, N. A vitamin D receptor selectively activated by gemini analogs reveals ligand dependent and independent effects. Cell Rep. 2015, 10, 516-526.

(23) Ciesielski F, Rochel N, Moras D. Adaptability of the vitamin D nuclear receptor to the synthetic ligand gemini: remodelling the LBP with one side chain rotation. J Steroid Biochem Mol Biol 2007, 103, 235-242.

(24) Galson, D.L.; Roodman, G.D. Pathobiology of paget's disease of bone. J Bone Metab. 2014, 21, 85-98.

(25) Belorusova, A.Y.; Eberhardt, J.; Potier, N.; Stote, R.H.; Dejaegere, A.; Rochel, N. Structural insights into the molecular mechanism of vitamin D receptor activation by lithocholic acid involving a new mode of ligand recognition. J Med Chem. 2014, 57, 4710-4719.

(26) Kabsch, W. Software XDS for image rotation, recognition and crystal symmetry assignment. Acta Crystallogr D Biol Crystallogr. 2010, 66, 125-132.

(27) Collaborative Computational Project, N. 4. The CCP4 suite: programs for protein crystallography. Acta Crystallogr. Sect. D Biol. Crystallogr. 1994, 50, 760-763.

(28) Evans, P. Scaling and assessment of data quality. Acta Crystallogr. D. Biol. Crystallogr. 2006, 62, 72-82. 
(29)Bricogne G, Blanc E, Brandl M, Flensburg C, Keller P, Paciorek W, Roversi P, Sharff A, Smart OS, Vonrhein C, Womack TO. BUSTER version 2.11.2. Cambridge, United Kingdom: Global Phasing Ltd 2011.

(30) Afonine PV, Grosse-Kunstleve RW, Adams PD. CCP4 Newsl 2005; 42, contribution 8.

(31) Emsley, P.; Cowtan, K. Coot: model-building tools for molecular graphics. Acta Crystallogr 2004, D6o: 2126-2132.

(32) Stephens, A.D.; Nespovitaya, N.; Zacharopoulou, M.; Kaminski, C.F.; Phillips, J.J.; Kaminski Schierle, G.S. Different Structural Conformers of Monomeric $\alpha$-Synuclein Identified after Lyophilizing and Freezing. Anal Chem. 2018, 90, 6975-6983.

(33) Rochel, N.; Ciesielski, F.; Godet, J.; Moman, E.; Roessle, M.; Peluso-Iltis, C.; Moulin, M.; Haertlein, M.; Callow, P.; Mély, Y.; Svergun, D.I.; Moras, D. Common architecture of nuclear receptor heterodimers on DNA direct repeat elements with different spacings. Nat Struct Mol Biol. 2011, 18, 564-570.

(34) Hourdel, V.; Volant, S.; O'Brien, D.P.; Chenal, A.; Chamot-Rooke, J.; Dillies, M.A.; Brier, S. MEMHDX: an interactive tool to expedite the statistical validation and visualization of large HDX-MS datasets. Bioinformatics. 2016; 32, 3413-3419. 


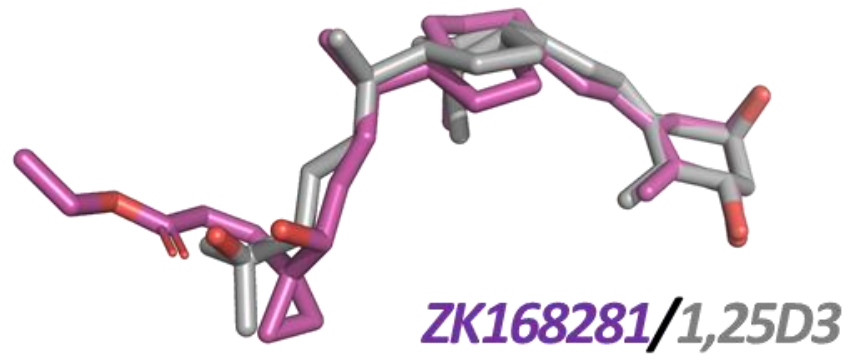

